\title{
Carbon Nanotube Reinforced Ceramic Matrix Composites- A Review
}

\author{
Subhranshu Sekhar Samal* and Smrutisikha Bal
}

Department of Metallurgical \& Materials Engineering

N.I.T Rourkela, Orissa, India-769008

* Corresponding Author:

Subhranshu Sekhar Samal

[Presently working as scientist in centre for Nanoscience \& Nanotechnology (A Joint

Initiative of $S U \& I G C A R)$, Chennai]

Ph: +91-9962369727, E-mail: sekhar_nitrkl@rediffmail.com

\section{ABSTRACT}

This paper provides an overview of recent advances reported in literature in ceramics matrix composites research in the context of reinforcement with carbon nanotubes. Current state of research has indicated the potential of these nanocomposites but at the same time, has illustrated the significant challenges in processing and improving properties. Fundamental work in processing, characterization and analysis is important before the structural properties of this new class of nanocomposites can be optimized. The fields of the nanocomposite materials have received a lot of attention and close scrutiny of scientists and engineers in recent years. This scrutiny results from the simple premise that using building blocks with dimensions in the nanometer range makes it possible to design and create new materials with unprecedented flexibility. The driving force behind the fabrication of novel nanocomposites is to achieve high functional properties for high end applications.

Key Words: reinforcement, nanocomposites, carbon nanotube, functional, ceramic matrix 


\section{INTRODUCTION}

Since the discovery of CNTs, they have been the focus of frontier research. It has opened vast areas of research which also include nanoscale reinforcements in composites in order to improve their mechanical, thermal and even electrical properties. Although the focus of the research in nanotube based composites has mostly been on polymer based composites, the unique properties of carbon nanotubes can also be exploited in ceramic matrix composites. But even after a decade of its discovery, the full potential of carbon nanotubes in this application has not been realized with experimental outcomes falling short of predicted values which demand an active insight in this field. Although ceramics have high stiffness and excellent thermal stability with low density, their brittleness impedes the use as structural materials. Because of their exceptional resilience, carbon nanotubes might be desirable as reinforcement for ceramics. The combination of these nanotubes with a ceramic matrix could potentially create composites that have high temperature stability as well as exceptional toughness and creep resistance. Also increased thermal and electrical conductivity of the composites even at low volume fractions might provide the thermal transport needed to reduce material operating temperatures and improve thermal shock resistance in applications like thermal elements and electrical igniters. The high temperature and high reactive environment during conventional fabrication methods of ceramics damage the carbon nanotubes and thus, there is a need of alternate methods of processing the composites. Also, there is also a need of control of interface between nanotube and the matrix for better interfacial bonding. But the research is still in embryonic stage and there are a lot of challenges to be resolved before it is ready for use in varied industrial applications. This review addresses the current progress of research in this field and the critical issues of research are

\section{INTRODUCTION TO CARBON NANOTUBES}

Understanding the basic science and technology of carbon nanotubes [1] can be very useful for better insight into the problem statement. Starting with the fundamentals, the stunning discovery of fullerenes by Nobel laureates Dr. Richard Smalley, Dr. Harold Kroto and Dr. Robert Curl in the year 1985 opened a new area of carbon chemistry. Carbon nanotubes were first observed under an electron microscope by Sumio lijima and his co-workers. It has been the object of intense scientific research ever since.

Carbon nanotubes can be visualized as a graphene sheet that has been rolled into a tube with hemispherical caps at both ends. Unlike diamond, where a 3-D diamond cubic crystal structure is formed with each carbon atom having four nearest neighbours arranged in a tetrahedron, graphite is formed as a 2-D sheet of carbon atoms arranged in a 
hexagonal array. In this case, each carbon atom is linked to three nearest neighbours. Rolling the sheets of graphite into cylinders form carbon nanotubes [2]. The properties of nanotubes depend on atomic arrangement (how the sheets of graphite are 'rolled'), the diameter and length of the tubes.

Nanotubes exist as either single-walled or multi-walled structures. Multi-walled nanotubes (MWNTs) are simply composed of concentric single-walled nanotubes (SWNTs) [Fig.1 (a\&b)]. Primary synthesis methods to prepare single walled and multi walled nanotubes include methods of arc discharge, laser ablation, gas phase catalytic growth from carbon monoxide and similar carbon sources and chemical vapour deposition. Considering the application of carbon nanotubes as reinforcements in composites which requires production of large amount of carbon nanotubes economically, gas phase techniques like chemical vapour deposition (CVD) offers the greatest potential for optimization of nanotube production.

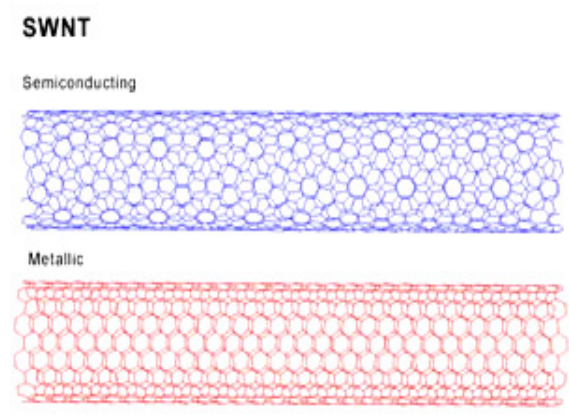

Fig 1(a): Single walled.

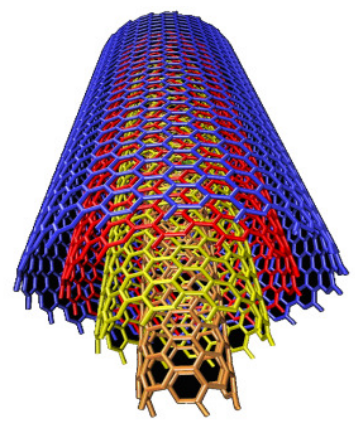

Fig 1(b): Multi-walled nanotube.

During nanotube synthesis, a lot of bi-products are also formed which include amorphous carbon, carbon black, non tubular fullerenes and also excess catalyst particles. This accounts for subsequent purification steps to separate and isolate nanotubes from the rest. Since the discovery of carbon nanotubes (CNT) in 1991 and the realization of their unique physical properties, including mechanical, thermal, and electrical, many investigators have endeavored to fabricate advanced CNT composite materials that exhibit one or more of these properties. To explore the full potential of carbon nanotubes for application in ceramic nanocomposites, one must fully understand the fracture and elastic properties of carbon nanotubes as well as the interactions at nanotube-matrix interface [3].

The multi-walled carbon nanotubes with a diametrical range of 5-40 $\mathrm{nm}$ are known for their exceptional mechanical properties. MWNT whose modulus is comparable to that of diamond (1.2 TPa), are reported to have strengths 10- 100 times higher than the strongest 
steel at a fraction of the weight [4]. This, coupled with approximately 500 times more surface area per gram (based on equivalent volume fraction of typical carbon fiber) and aspect ratios of around 1000, has created a great deal of interest in using CNT as a reinforcing phase for polymer matrices.

In addition to the exceptional mechanical properties, they also possess superior thermal and electrical properties. They have a thermal stability up to $750^{\circ} \mathrm{C}$ in air and $2800^{\circ} \mathrm{C}$ in vacuum. Their thermal conductivity is about twice as high as diamond, with electric current transfer capacity 1000 times greater than copper wires. For high-temperature applications, the high thermal conductance of CNTs suggests that their incorporation, even at low volume fractions, might provide the thermal transport needed to reduce material operating temperatures and improve thermal shock resistance.

Electrical conductance can also be useful in ceramic-based materials, as heating elements and electrical igniters, for instance. For example, as conductive fillers, CNT are quite effective compared to traditional carbon black micro particles, primarily due to their high aspect ratios. The electrical percolation threshold was recently reported at 0.0025 wt. \% $\mathrm{CNT}$ and conductivity at $2 \mathrm{~S} / \mathrm{m}$ at $1.0 \mathrm{wt} \% \mathrm{CNT}$ in epoxy matrices. Control of electrical resistance through the use of CNT as filler is therefore, an attractive alternative to other composite systems [5]. These exceptional properties have been investigated for devices such as field emission displays (FED), scanning probe microscopy tips, and microelectronic devices.

Although single walled nanotubes possess better properties than multiwalled nanotubes, but the relative simplicity in preparation of multiwalled nanotubes and ease in its isolation and purification makes it more economical for most applications including dispersing in ceramic matrix. Another property that is getting a lot of attention is the nanotubes surface, namely the interface between the carbon nanotubes and surrounding composite matrix. From micromechanics, it is through shear stress build-up at this interface that stress is transferred from the matrix to the nanotubes. Numerous researchers have attributed lower-than predicted carbon nanotube-ceramic and carbon nanotubepolymer composite properties to a lack of interfacial bonding. If one considers the surface of a carbon nanotube, essentially an exposed graphene sheet, it is not surprising that interfacial interaction is a concern. It is the weak inter-planar interaction of graphite that provides its solid lubricant quality, and resistance to matrix adhesion. If the mechanical properties of multi walled nanotubes can be effectively incorporated into a ceramics matrix, comparatively lighter weight composites with exceptional strength can be achieved. In ceramic matrix materials, the mechanical property of greatest importance is toughness or resistance to crack growth. Ceramics are already stiff and strong and so, in analogy to ceramic composites with micron-scale fiber additions, the incorporation of carbon nanotubes is aimed at enhancing toughness. NASA (USA) is focusing on making 
use of CNTs to develop high-strength composite materials for the next generation spacecraft via this revolutionary technology.

\section{CERAMIC NANOCOMPOSITES}

The field of nanocomposite materials has received a lot of attention and close scrutiny of scientists and engineers in recent years. This scrutiny results from the simple premise that using building blocks with dimensions in the nanometer range makes it possible to design and create new materials with unprecedented flexibility. The driving force behind the fabrication of novel nanocomposites is to achieve high functional properties for high end applications. Nanocomposites can be found in nature in the form of biological systems, such as in plants and bones. Nanocomposites can be considered solid structures with nanometer-scale dimensional repeat distances between different phases that constitute the structure. These materials typically consist of an inorganic (host) solid containing an organic phase or vice versa. Or they can be consisting of two or more inorganic or organic phases in some combinatorial form with the constraint that at least one of the phases or features is in the nanometer size. Extreme examples of nanocomposites can be porous media, colloids, gels and copolymers. The nanostructured phase can be 0-D (embedded clusters), 1- Dimensional (Carbon nanotubes), 2-Dimensional (nanoscale coatings) or 3- Dimensional (embedded networks). In general, nanocomposite materials can demonstrate different mechanical, electrical, optical, electrochemical, catalytic and structural properties than those of individual components. The multifunctional behavior of any specific property of the material is often more than the individual sum of the individual components.

The term and the concept 'nanocomposite' were formally adopted for ceramic materials by Roy, Komarneni and colleagues about a decade ago. They developed hybrid ceramic and metal nanocomposite materials synthesized by sol-gel processes and discussed quasicrystalline one- or two- component materials, with isolated phases or regions of 1-10 nm with different structure and/or composition. The concept of structural ceramic nanocomposites was proposed by Niihara in 1991 and can be seen as an adoption of the nanocomposite approach for the micro structural tailoring of structural ceramic composites. This work was mainly based on results obtained on the $\mathrm{Si}_{3} \mathrm{~N}_{4} / \mathrm{SiC}$ and $\mathrm{Al}_{2} \mathrm{O}_{3} / \mathrm{SiC}$ systems [6]. Photosensitive glasses which show precipitation of silver halogenides might be the most common application of the nanocomposite approach. Many efforts are underway to develop high-performance ceramics that can withstand conditions of high temperature, wear and chemical attack as required in numerous advanced applications like highly efficient gas turbines, aerospace materials, automobiles, etc. 
Most ceramic materials pose some grave problems, including relatively low fracture toughness and strength, degradation of mechanical properties at high temperatures, and poor resistance to creep, fatigue and thermal shock. Attempts to solve these problems have involved incorporating second phase such as particulates, platelets, whiskers, and fibers in the micron-sized range at the matrix grain boundaries. But these problems are not solved effectively with micron-sized fillers. The passive control of the microstructures by incorporating nanometer-size second phase dispersions into ceramic matrices has given a promise in this direction.

There are only a few concepts available to compensate for the disadvantage of brittleness in ceramics and to improve the strength. Following Griffith's approach, the fracture strength of brittle materials can only be improved by an increase in fracture toughness or by a reduction in critical flaw size. Therefore, much effort has been invested in sophisticated processing technology to reduce the size and density of processing flaws. The design of tougher, flaw tolerant ceramics is a more interesting approach for wider industrial applications since it improves the reliability of a component. The fracture toughness can be increased by incorporating various energy dissipating components into the ceramic microstructure. These components can be inclusions such as whiskers, platelets or particles of microns size. The reinforcements serve to deflect the crack or to provide bridging elements hindering further opening of the crack. Another concept is to incorporate metallic ligaments into the ceramic matrix to form crack bridging elements that absorb energy by plastic deformation. Finally, much benefit has resulted from incorporating a second phase which undergoes a phase transition in conjunction with a volume expansion initiated by the stress field of a propagating crack; this, too, can apply a closing force on the crack.

The most promising solution which is currently researched extensively is the dispersion of nanoparticles, especially carbon nanotubes into the ceramic matrix. Apart from structural applications, nanocomposite technology can also be applied for fabricating functional ceramics like ferroelectric, piezoelectric, varistor and ion conducting materials. Incorporating a small amount of ceramic and metallic nanoparticles into Barium Titanate, Zinc Oxide or cubic Zirconia can significantly improve their strength, hardness, and toughness, which are very important in creating highly reliable electric devices operating in severe environmental conditions. In addition, dispersing conducting metallic nanoparticles or nanowires can enhance the electrical properties. For example, the range of electroceramic nanocomposites for information and charge storage has revolutionized the electronic industry. In the nanoscale, quantum effects can be utilized to modify energy states and electronic structures of components. Conducting nanosized particles dispersed in a dielectric matrix (i.e. Ni in PZT) can improve dielectric properties. Unique catalytic properties can be achieved with entrapment- type nanocomposites where, for 
example, fine metal clusters are supported in zeolites (three-dimensionally linked network structures with channels up to $1 \mathrm{~nm}$ ).

The solution sol-gel technique, in particular, offers an opportunity to produce not only ultra-homogeneous materials but also heterogeneous or nanocomposite materials. After crystallization and densification those materials are appropriate for numerous applications, such as electronic or structural materials.

The most common methods for processing nanocomposites are mechanical alloying, sol gel synthesis, and by thermal spray synthesis. Mechanical alloying occurs as a result of repeated breaking up and joining of the component particles which can prepare highly metastable structures such as amorphous alloys and nanocomposite structures with high flexibility. Scaling up of synthesized materials to industrial quantities is easily achieved in this process, but purity and homogeneity of the structures produced remains a challenge. In addition to the erosion and agglomeration, high-energy milling can provoke chemical reactions that are induced by the transfer of mechanical energy, which can influence the milling process and the properties of the product. This is used to prepare magnetic oxide-metal nanocomposites via mechanically induced displacement reactions between a metal oxide and a more reactive metal.

Sol-gel is another process in which aerogels are the precursors. Aerogels are made by solgel polymerization of selected silica, alumina or resorcinol-formaldehyde monomers in solution and are extremely light but very porous, mostly nano-sized pores. In the nanocomposites derived from aerogels, the product consists of a substrate like silica gel and one or more additional phases with one of the phases with dimension of the order of nanometers.

Thermal spray coating is another commercially relevant, proven technique for processing nanostructured coatings. Thermal spray techniques are effective because agglomerated nanocrystalline powders are melted, accelerated against a substrate and quenched very rapidly in a single step. This rapid melting and solidification promotes retention of a nanocrystalline phase and even amorphous structure. Retention of nanocrystalline structure leads to enhanced wear behavior, greater hardness, and sometimes a good coefficient of friction compared to conventional coatings.

As the research in nano-reinforced ceramics is still in its infancy, it is not clear which, if any, of the above toughening and strengthening mechanisms apply to ceramic nanocomposites. The aim of this review is to develop a rational path for the optimization of structural nanocomposites on the basis of current observations and consequent hypotheses. Particular emphasis will be given to common ceramic matrices containing 
carbon nanotubes as reinforcements because these systems have been the subject of the greatest study

\section{PROCESSING AND PROPERTIES OF CNT REINFORCED COMPOSITES}

The nanotube reinforcements promise to increase the fracture toughness of the composites by absorbing energy through their highly flexible elastic behaviour during deformation, which will be especially important for nanotube-based ceramic matrix composites. But, materials fabrication difficulties have limited research on nanotubereinforced ceramic composites. The major concern is to obtain a uniform dispersion of nanotubes in the matrix. Damage or destruction of the nanotubes is often observed because of the high temperatures and highly reactive environments associated with many methods of processing ceramic and metal matrices. Also lack of optimized fabrication and processing of nanotubes has also restricted research efforts, although this seems to have become less of a problem in recent years as more groups produce their own nanotubes with catalyzed chemical vapor deposition (CVD) methods.

Powder processing methods have been used to fabricate ceramic matrices. The small diameter and large aspect ratio of the nanotubes can make it difficult to obtain a good mixture of the two phases prior to sintering or hot pressing. Some success has been achieved with conventional milling techniques, primarily with the use of low to moderate nanotube volume fractions. Peigney et al. have successfully employed super plastic forming with mixtures of submicron powders and CNTs [7]. Here the nanotubes appear to facilitate the high-temperature extrusion process. This method produces a ceramic matrix composite (CMC) in which the nanotubes are highly aligned [Fig.2].

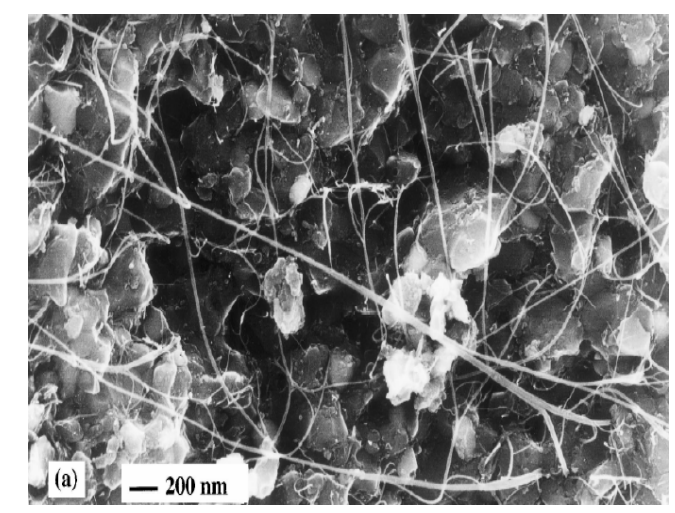

Fig 2: HREM micrograph of CNT-Magnesium Aluminide composite powders with highly aligned CNTs. 
Balazsi et al. have devised a novel processing method to make a composite material consisting of a highly durable $\mathrm{Si}-\mathrm{B}-\mathrm{N}-\mathrm{C}$ ceramic matrix made from substituted vinylborazine precursors modified by carbonaceous structures on the surface [8]. Depending on the content of $\mathrm{Ni}$ dopand of the starting polymer, a catalyzed growth of multiwalled carbon nanotubes (CNTs) of the bamboo-type or of carbon layers is observed [Fig.3 (a\&b)]. The presented new composite material with a thermal stability up to $1500{ }^{\circ} \mathrm{C}$ of both the carbonaceous or nanotube coatings and the ceramic matrix promises a wide range for novel applications. Especially, a strong immobilization of the CNTs allows using this material as a support for heterogeneous catalysts.

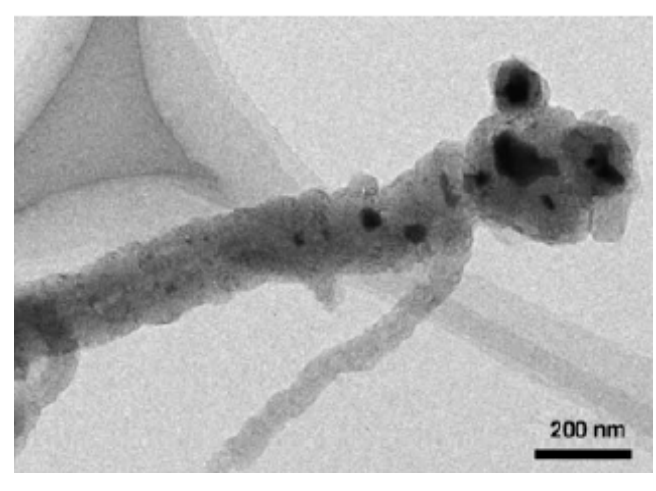

(a)

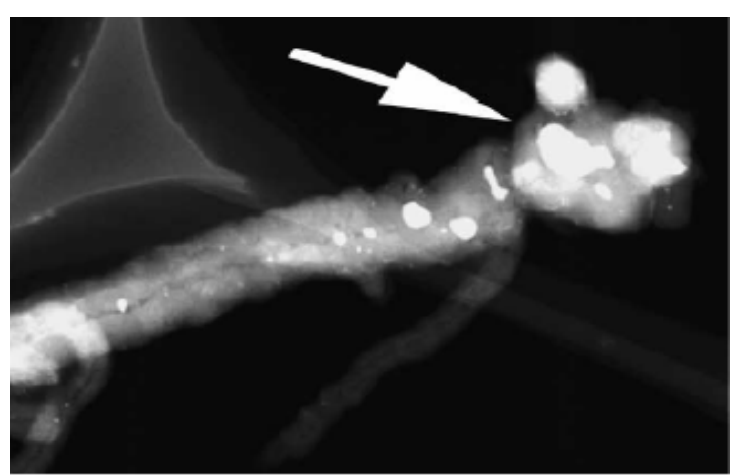

(b)

Fig 3 (a\&b): Selected carbon nanotube after Carbonyl Iron-impregnation as well as $\mathrm{Fe} \mathrm{Ni}$ exchange.

The functionalization of the CNTs with metals via common impregnation methods is demonstrated exemplarily. By minimizing the ceramic particle size, large surface carbon nanotube materials can be achieved. Ma et al. have successfully fabricated nano-SiC ceramic-based CNT composites by mixing SiC nanoparticles with 10 wt. \% CNTs and hot pressing at $2273^{\circ} \mathrm{K}$. They found that the CNTs played a strengthening and toughening role in this ceramic matrix composite. Both bend strength and fracture toughness of this $\mathrm{SiC}-10 \%$ CNTs composite increase by about $10 \%$ over monolithic SiC ceramic.

Another interesting approach is to use a mixture of ceramic powder and catalyst for nanotube growth $(\mathrm{Fe}, \mathrm{Ni}$, or $\mathrm{Co})$, followed by a CVD process that grows nanotubes inside the particulate perform. This can produce a uniform mixture of the two phases as long as mass transport of the carbon-containing gas (e.g. $\mathrm{C}_{2} \mathrm{H}_{2}, \mathrm{CH}_{4}$, etc.) into the powder bed does not produce significant gradients. 
In some cases, the nanotubes are damaged by high temperature sintering. However, spark-plasma-sintering has been used to produce materials where the nanotube structure is retained [Fig.4]. The success of this method apparently results from the use of lower temperatures and shorter firing times. While the sintering of traditional whisker and fiber reinforced composites is typically plagued by differential densification rates, this may be less of a problem with nanotubes because of their small dimensions. Conventional hotpressing and hot isostatic pressing methods have also been used to produce nanotubereinforced composites. Tape casting and lamination method have been used to achieve better dispersion of CNTs in an alumina matrix. The tribological studies of the fabricated composites showed that tape casting helped in achieving higher dispersion. The presence of CNTs is also expected to inhibit grain growth during high-temperature processing. This beneficial effect is also known to occur during high-temperature processing of a variety of other composite materials.

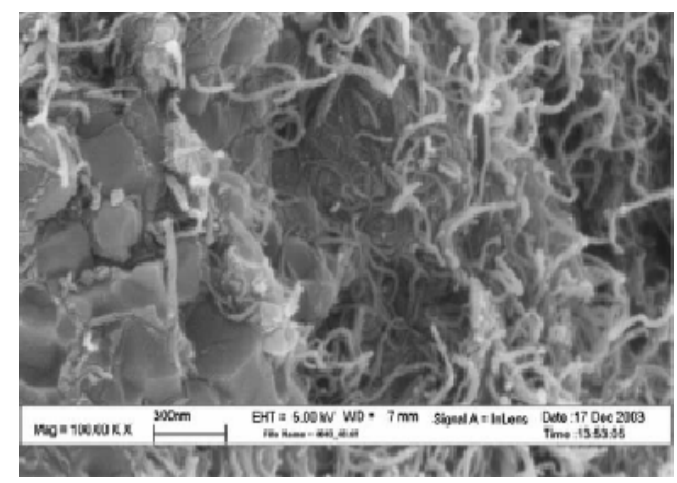

Fig 4: SEM images of CNT dispersed SiN composite prepared from SPS.

High temperature fabrication of a ceramic matrix can create some difficulty in identifying nanotubes in the final composite, particularly when low volume fractions and relatively small nanotubes are used. However, high-resolution scanning electron microscopy can often be used to image nanotubes directly. Raman spectroscopy is also a useful tool for characterizing the carbon bonding indicative of nanotubes. Several methods have been used to form nanotubes inside preexisting porous matrices. Rul et al. formed a porous spinel structure with gel casting [9]. This matrix also contained a small amount of Co catalyst, and subsequent CVD produced a relatively high nanotube volume fraction inside the porous oxide. Another method of producing nanotubes inside a porous ceramic is to use anodic alumina templates with controlled pore structures [Fig.5]. This approach is generally limited to coatings or membranes up to $\sim 100 \mu \mathrm{m}$ thick. The resulting materials consist of aligned nanotubes whose diameter is determined by the pre-existing cylindrical (or nearly cylindrical) pores in the alumina. The dimensions of these materials make them potentially applicable as coatings. Also, the unidirectional microstructure is an advantage for fundamental investigations of mechanical properties. 


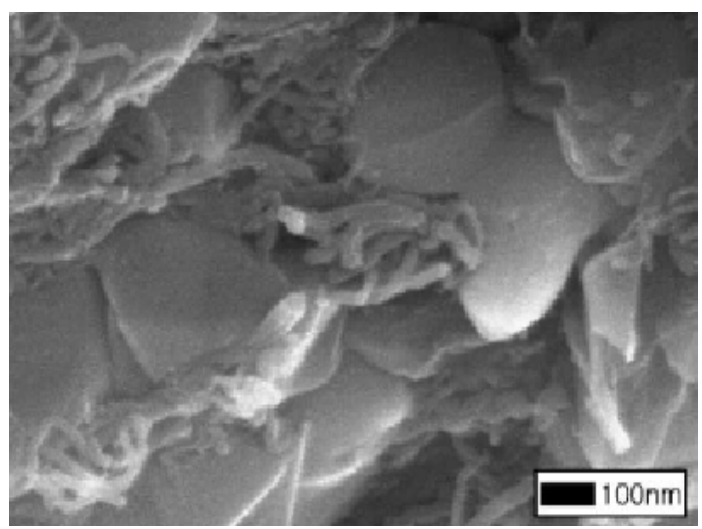

Fig. 5. Microstructure of CNT dispersed Alumina composite made by hot pressing technique.

Nanoscale powders with carbon nanotubes provide another opportunity for creating dense ceramic-matrix powders with enhanced mechanical properties. The strength and fracture toughness of hot pressed á-alumina is typically much greater than that of conventional grain size polycrystalline alumina. Addition of carbon nanotubes to the alumina results in lightweight composites with even greater strength and fracture toughness. The mechanical properties of such composites depend strongly on the processing methods and surface treatment of the carbon nanotubes. The work on characterization and the processing of the mechanical properties of a carbon nanotube-reinforced Al-based composite prepared by hot-pressing followed by hot-extrusion indicated that the carbon nanotubes in the composites are not damaged during the composite preparation and that no reaction products at the nanotube/Al interface are visible after annealing for $24 \mathrm{~h}$ at $983^{\circ} \mathrm{K}$. The strength of the composites is slightly affected by the annealing time at $873^{0}$ K.

The most interesting and challenging applications for CNT ceramic matrix composites (CNT/CMCs) are as tough materials which has seen bulk of research. Toughening in CMCs is typically achieved by a weak fiber/matrix interface coupling that permits debonding and sliding of the fibers within the matrix. The closing forces exerted by fibers on matrix cracks that propagate around the fibers constrains the crack growth, and the work required to pull broken fibers out against residual sliding friction at the fiber/matrix interface imparts significant fracture toughness to CMCs. Weak interfaces between a CNT and many ceramic matrices is expected, and pyrolytic carbon is the most common interface material deposited onto fibers to induce such debonding. Thus the mechanisms of interface debonding and sliding, and associated toughening should be operative in CNT-CMCs unless the sliding resistance is too low (see below).

Most work on CNT- CMCs has focused on the measurement of toughness using the indentation/crack-length technique, which is nominally suitable for small volumes of 
material, with little emphasis on uncovering actual mechanisms of any toughening. Unfortunately, most results for toughening have been disappointing. Data has shown very little or no increase in toughening upon introduction of CNTs - either single or multiwalled into various ceramic matrices. Zhan et al however, have reported significant fracture toughness improvements, three times higher than an unreinforced matrix, based on indentation measurements [10]. On the other hand, new results from Wang et al. demonstrate that the indentation/crack-length technique is not valid in these materials. Wang et al. fabricated CNT-alumina materials using the method of Zhan et al., but performed the far more reliable single edge V-notched beam test, which showed no enhanced toughening and thus refutes the claims of high toughness by Zhan et al. To reinforce their conclusion, Wang et al. performed indentation tests on the CNT- CMCs and on composites containing only graphite particles and showed that the indentation behavior is similar in the two materials: cracking is suppressed so that the 'apparent' toughness is high [11]. These works show that careful measurements are required and also strongly suggest that indications of enhanced toughening should be supported by direct evidence of potential toughening mechanisms. Although not tough in a traditional sense, the CNT-CMCs and graphite-CMCs are 'damage tolerant' under contact loading [Fig.6 (a\&b)], i.e. they do not crack easily, which is explained by noting that the carbon additions may accommodate deformations by shear under the indenter. New work by Xia et al. has emphasized the identification of toughening mechanisms, rather than the determination of numerical values for toughness [12]. They used a novel CNT-CMC material consisting of an aligned, ordered array of (imperfect) multiwalled CNTs embedded in an alumina matrix. The interaction of cracks, induced by nanoindentation, with the nanotubes could be directly observed for several orientations. The key phenomena of crack deflection at the matrix/nanotube interface crack bridging by nanotubes [Fig.7], and nanotube pull-out on the fracture surface all indicate that appropriate toughening mechanisms can operate in a CNT-CMC. They also show direct evidence of a novel shear deformation mode that is consistent with the arguments of Wang et al. regarding 'damage tolerance' driven by shear. Further work on such model systems, as well as more practical systems, is needed to establish quantitative toughening levels, however.

Other mechanical properties, such as hardness and elastic modulus, have been investigated as well, with inconclusive results. CNT additions to silicon nitride show some variation in strength and modulus, but the CNTs change the density of the material and the strongest correlation in mechanical properties is with density. Investigations on CNT alumina materials showed an increase in hardness and decrease in wear resistance for moderate CNT additions, say 2-4\%. However, the CNTs change the grain size of the resultant matrix and the hardness correlates with the grain size changes as well. These results demonstrate that processing-induced changes in the matrix may have greater 
effects on mechanical properties than the actual presence of CNTs. Thus, correlations of properties solely with CNT content can be highly misleading.

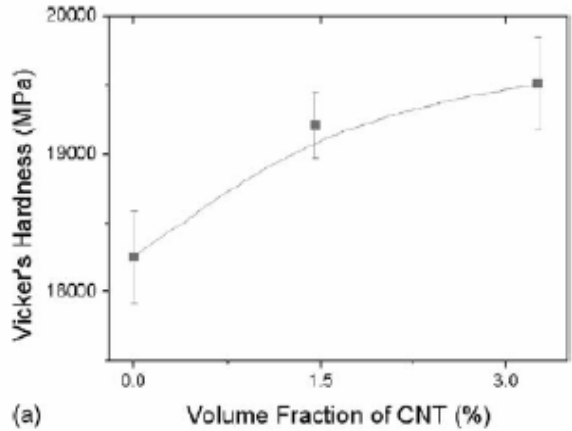

(a)

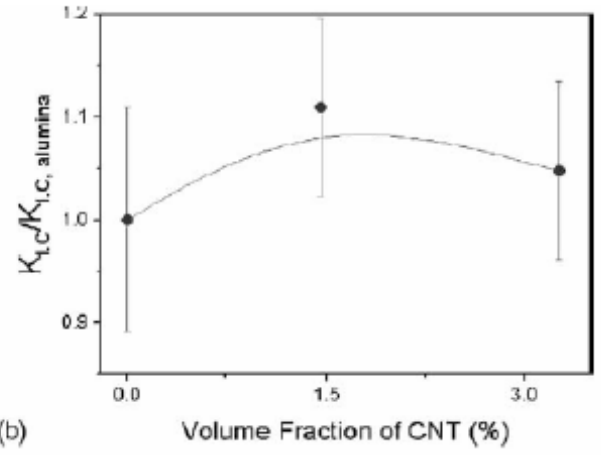

(b)

Fig 6: Representation of hardness (a) and fracture toughness (b) as a function of CNT \%.

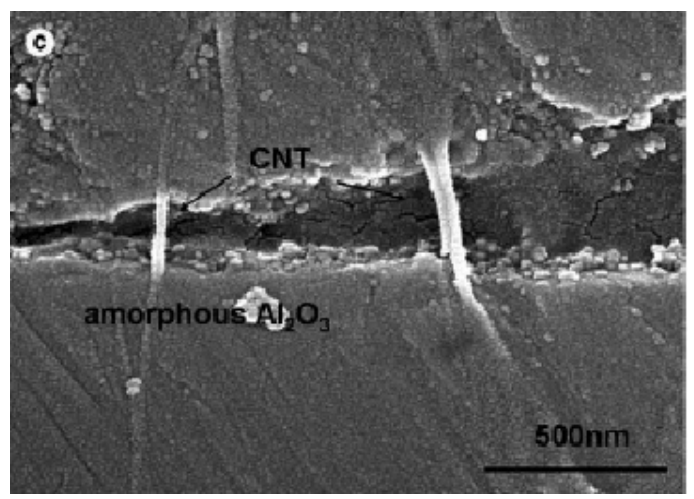

Fig 7: Crack Bridging Mechanism.

CNT-CMC materials have demonstrated significant enhancements in electrical conductivity. Physically long, thin, and electrically conducting nanotubes create an interconnected percolating network of conducting paths through an otherwise insulating matrix to yield high conductivity at relatively low CNT volume fraction [13].

\section{CONCLUSIONS AND FUTURE SCOPES}

CNT-based composite systems continue to hold promise but with significant challenges to real success. Given the costs of the materials and processes involved, it is not sufficient to obtain marginal improvements in properties over traditional micron-scale composites or virgin matrices. Yet, with few exceptions, notable enhancements have not been 
observed. The traditional interplay of careful processing and evaluation, coupled with mechanistic assessment of properties, remains a valid paradigm at the nanoscale and should be assiduously applied to future research in CNT composite systems.

The work reviewed here demonstrates that a variety of methods for producing ceramic and metal matrix nanotube composites have been investigated in recent years, but with mixed results on mechanical performance. Further research on these approaches will almost certainly lead to better materials.

Better mechanical properties are likely to result from higher nanotube volume fractions and, in some cases, the use of longer nanotubes. Both of these variations create additional processing challenges because it is more difficult to obtain a uniform dispersion of nanotubes and, in some cases, more difficult to obtain a dense matrix.

A key issue that has not yet been explored in any detail is the control of the interface between the nanotubes and the matrix. Experience with other composites indicates that these interfaces will be critical for mechanical performance.

In particular, it is well established that debonding and interfacial sliding are critical in conventional CMCs. In a CNT-reinforced material, interface behavior is expected to be significantly different from conventional carbon fibers because of the unique chemical bonding inherent in CNTs. Experiments have shown that high-quality, multiwalled CNTs exhibit very easy interwall sliding, with inner graphitic walls able to be extracted from outer walls in a 'sword and sheath' mechanism at low applied forces. Moreover, this behavior is not frictional in nature - the pull-out forces do not scale with embedded length.

Molecular dynamics simulations have shown that the pull-out forces are increased when the inner walls have a fractured end. The fractured end of the inner wall(s) would naturally deform outward but is constrained by the outer wall(s), generating high local stress, requiring pull-out forces four times larger than capped-end nanotubes, consistent with experiments. The behavior is still not frictional, however, and incorporation of nearly perfect CNTs may not be the appropriate direction for design of high-performance materials.

The behavior obtained on imperfect nanotubes, for instance may suggest that nanocarbon fibers rather than nanotubes hold most promise for providing the balance of properties (strength and interfacial sliding behavior) needed for good mechanical performance. The smaller length scales associated with nanotubes will also create differences in comparison with traditional composites. For ceramic composites with micronsized carbon or ceramic fibers, the interface properties are typically controlled with a layer of pyrolytic carbon or boron nitride that is at least several hundred nanometers thick. An analogous, 
proportionally sized layer for CNTs would potentially involve control of nanotube-matrix bonding at or near the atomic length scale.

It is also important to note that work done to date has utilized both single- and multiwalled nanotubes with a variety of different structures, with the choice of CNT material often dictated by availability or processing considerations. For example, in situ methods based on CVD at moderate temperatures typically produce multiwalled nanotubes with a relatively disordered structure. It is not yet clear how these different nanotube structures will affect mechanical behavior, and processing issues associated with both nanotube degradation and interfacial bonding are likely to depend, at least in part, on the structure of the nanotubes. Properties that do not rely on high synergy between the matrix and CNTs, such as electrical conductivity, have already shown notable enhancements. The synergy attending other electromagnetic behaviors in CNT-metal systems is encouraging, but not yet fully studied.

\section{REFERENCE}

[1]S. Iijima, "Helical microtubules of graphitic carbon", Nature, 1991, v354, pp. 56-58. [2] P. J. F. Harris, 'Carbon nanotubes and related structures', Cambridge University press, 1999.

[3] E. T. Thostenson, Zhifeng Ren, Tsu-Wei Chou, "Advances in the science and technology of carbon nanotubes and their composites”, Composite Science and Technology, 2001, v61, pp. 1899-1912.

[4] Young-Hag Koh, Hae-Won Kim and Hyoun-Ee Kim, “Mechanical behavior of polymer and ceramic matrix nanocomposites”, Scripta Materialica, 2001, 44 v8-9, pp. 2061.

[5] E. Flahaut, A. Peigney, Ch. Laurent, Ch. Marlière, F. Chastel and A. Rousset, "Carbon nanotube-metal-oxide nanocomposites: microstructure, electrical conductivity and mechanical properties”, Acta Materialia, 2000, v48, pp. 3803-3812.

[6] J. W. An, D. H. You and D. S. Lim “Tribological properties of hotpressed alumina/CNT composites”, Wear 2003, v255, pp.677-681.

[7] A. Peigney, E. Flahaut, Ch. Laurent, F. Chastel and A. Rousset, "Aligned carbon nanotubes in ceramic-matrix nanocomposites prepared by hightemperature extrusion", Chemical Physics Letters, 2002, v352, pp. 20-25.

[8] Balazsi, Z. Kónya, F. Wéber, L. P. Biró and P. Arató, “Preparation and characterization of carbon nanotube reinforced silicon nitride composites”, Materials Science Engineering C, 2003, v23, pp. 1133-1137.

[9] S. Rul, Ch. Laurent, A. Peigney and A. Rousset, "Carbon nanotubes Prepared in situ in a cellular ceramic by the gelcasting-foam method", Journal of the European Ceramic Society, 2003, v23, pp. 1233-1238. 
[10] Guo- Dong Zhan, Joshua Kuntz, Julin Wan, and A.K. Mukherjee, "Singlewall carbon nanotubes as toughening agents in alumina-based nanocomposites", Applied Physics Letters. 2003, v83, pp.1228-1233.

[11] Xiaotong Wang, Nitin Padture and Hidehiko Tanaka, "Contact damageresistant ceramic/single-wall carbon nanotubes and ceramic/graphite composites", Nature Materials, 2004, v3, pp. 539-544.

[12] Z. Xia, L. Riester, W. A. Curtin, H. Li, B. W. Sheldon, J. Liang, B. Chang and J. M. $\mathrm{Xu}$, "Direct observation of toughening mechanisms in carbon 23 nanotube ceramic matrix composites”, Acta Materialia, 2004, v52, pp. 931-944.

[13] S. Rul, F. Lef_evre-schlick, E. Capria, Ch. Laurent, A. Peigney, "Percolation of single-walled carbon nanotubes in ceramic matrix nanocomposites”, Acta Materialia, 2004, v52, pp. 1061-1067. 